\title{
Efficient generation of Rosa26 knock-in mice using CRISPR/Cas9 in C57BL/6 zygotes
}

\author{
Van Trung Chu $^{{ }^{* *}}$, Timm Weber $^{1 \dagger}$, Robin Graf ${ }^{1 \dagger}$, Thomas Sommermann ${ }^{1}$, Kerstin Petsch ${ }^{1}$, Ulrike Sack ${ }^{2}$, \\ Pavel Volchkov ${ }^{3}$, Klaus Rajewsky ${ }^{1}$ and Ralf Kühn ${ }^{1,4^{*}}$
}

\begin{abstract}
Background: The CRISPR/Cas9 system is increasingly used for gene inactivation in mouse zygotes, but homologydirected mutagenesis and use of inbred embryos are less established. In particular, Rosa26 knock-in alleles for the insertion of transgenes in a genomic 'safe harbor' site, have not been produced. Here we applied CRISPR/Cas9 for the knock-in of 8-11 kb inserts into Rosa26 of C57BL/6 zygotes.

Results: We found that 10-20\% of live pups derived from microinjected zygotes were founder mutants, without apparent off-target effects, and up to $50 \%$ knock-in embryos were recovered upon coinjection of Cas9 mRNA and protein. Using this approach, we established a new mouse line for the Cre/loxP-dependent expression of Cas9.

Conclusions: Altogether, our protocols and resources support the fast and direct generation of new Rosa26 knock-in alleles and of Cas9-mediated in vivo gene editing in the widely used C57BL/6 inbred strain.
\end{abstract}

Keywords: CRISPR, Cas9, Knock-in mice, Rosa26, Zygotes

\section{Background}

The Rosa26 locus on chromosome 6 is frequently used for the integration of transgene constructs to achieve ubiquitous or conditional gene expression in mice. The Rosa26 transcript is spliced into three exons and ubiquitously expressed in all cell types and developmental stages, but not translated to a protein [1]. The locus was first identified by the integration of the Rosa $\beta$-geo (reverse orientation splice acceptor $\beta \mathrm{Gal}$ ) gene trap vector in pool \#26 of transduced embryonic stem (ES) cells [2]. This integration site, residing at the XbaI site within the first intron of Rosa26, has been used for ES-based gene targeting from its discovery on. A Rosa 26 targeting vector is extending $1 \mathrm{~kb}$ upstream and $4 \mathrm{~kb}$ downstream from the integration site within the first intron, flanking transgene inserts [3]. In the classical gene targeting procedure, targeted ES cell clones are injected into blastocysts to obtain germline chimeric mice and the transmission of targeted alleles to their offspring. This approach requires laborious handling of ES cell cultures and waiting times of 9-12 months until identification of positive $F_{1}$ pups [4]. Nevertheless, the

\footnotetext{
* Correspondence: VanTrung.Chu@mdc-berlin.de; ralf.kuehn@mdc-berlin.de ${ }^{\dagger}$ Equal contributors

${ }^{1}$ Max-Delbrück-Center for Molecular Medicine, 13125 Berlin, Germany Full list of author information is available at the end of the article
}

Rosa26 locus is frequently targeted via ES cells for inserting single transgene copies in a standardized configuration into the mouse genome. The Mouse Genome Informatics database (MGI, www.informatics.jax.org) refers to 562 Rosa26 knock-in mouse strains that have been generated for probing the effects of constitutively or conditionally expressed mutant proteins or for the imaging of reporter genes in vivo. Rosa 26 knock-in alleles are often configured such that coding regions are expressed under the control of the CAG hybrid promoter [5] or they are connected with splice acceptor elements to the endogenous Rosa26 transcript [3]. Conditional gene expression is achieved by insertion of a loxP-flanked transcriptional stop element between the promoter and coding regions. In such a case, gene expression is induced by crossing the conditional knock-in line with transgenic mice expressing Cre recombinase in specific cell types [6].

Double-strand breaks (DSB) induced by engineered nucleases in mouse zygotes have emerged as powerful tool for the direct, single step production of targeted mutants, independent of ES cells. Proof of principle was provided with Zinc-finger nucleases and TALENs [7, 8], both of which have been largely displaced by the more versatile and efficient CRISPR/Cas9 gene editing system [9]. This system is composed of the generic Cas9 nuclease 
that is guided to specific target sites by short sgRNAs including 20 nucleotides complementary to the target sequence upstream of a PAM signal (NGG). Gene editing is achieved by endogenous DSB repair pathways, either imprecisely by non-homologous end joining (NHEJ) causing small deletions, or by homology-directed repair (HDR) using repair template vectors for the precise insertion of new sequences. In mouse zygotes, CRISPR/Cas9 has been efficiently used for generating small deletions and knockout mutations by the NHEJ repair pathway, reaching frequencies of $50 \%$ in pups derived from RNA microinjections $[10,11]$, even in inbred backgrounds such as $\mathrm{C} 57 \mathrm{BL} / 6$. In contrast, HDR events with co-injected targeting vectors occur rarely in zygotes. A limited number of studies reported the generation of knock-in alleles at frequencies of 5-15\% for a small number of genes $[11,12]$, not targeting Rosa26 and using genetic hybrid embryos known for superior viability. Thus, an approach for the direct production of Rosa26 knock-in alleles in C57BL/6 embryos is presently not established, despite this inbred background being a standard in biomedical research.

Here we applied CRISPR/Cas9 for the knock-in of conditional transgenes into Rosa26 of C57BL/6 zygotes. Using modified Cas 9 mRNA and sgRNA targeting the intronic XbaI site of Rosa26, compatible with common targeting vector homology regions, we achieved the knock-in of $8-11 \mathrm{~kb}$ inserts in $10-20 \%$ of pups derived from microinjections of C57BL/6 embryos. This frequency increased to $50 \%$ upon the combined microinjection of Cas9 mRNA and Cas9 protein, as tested in blastocyst assays. In addition to editing of the mouse germ line in zygotes, CRISPR/Cas9 offers a new perspective for modifying gene function in somatic tissues. To avoid the vector-mediated delivery of the large Cas9 transgene into primary cells, we generated Rosa26 knock-in mice for the Cre/loxP-dependent expression of Cas9. Taken together, our protocols and resources support the fast and direct generation of new Rosa26 knock-in alleles and of Cas9-mediated in vivo gene editing in the C57BL/6 background.

\section{Results}

\section{Efficient DSBs induction at the Rosa26 intronic Xbal site in C57BL/6 zygotes}

To achieve CRISPR/Cas9-mediated knock-in into Rosa26, we selected sgRNA target sequences spanning the XbaI site within the first intron, adapted to the homology regions of gene targeting vectors used for ES cells that cover sequences up- and downstream of this site [3]. As we have shown previously, sgRosa26-1 (Fig. 1a) exhibits high activity in mouse cells [13]. We therefore selected sgRosa26-1, together with a Cas9 mRNA that includes a plasmid coded polyadenine (polyA) tail (Cas9-162A) [14], for targeting in zygotes. The most effective concentrations of Cas9-162A and sgRosa26-1 RNAs were determined by microinjection of varying amounts of RNA into the pronuclei of C57BL/ 6 zygotes, followed by embryo culture to the blastocyst stage. Genomic DNA was extracted from each blastocyst and used for PCR amplification of the target region (Fig. 1b). PCR products were analyzed for NHEJ repair-associated deletions by digestion with $\mathrm{XbaI}$ or the T7 endonuclease I (T7EI). At the lowest concentrations of

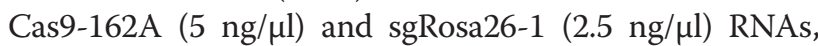
Rosa 26 alleles from $40 \%$ of the embryos exhibited sequence deletions, as shown by the presence of XbaI resistant bands, whereas T7EI assays were less sensitive (Fig. 1c). Sequencing of cloned PCR products from four blastocysts confirmed the presence of small deletions at the expected cleavage site. Of note, individual deletion events could generate new XbaI sites, causing an underestimation of gene editing events by $\mathrm{XbaI}$ digestion (Fig. 1d). Upon RNA microinjection of Cas9-162A at $25 \mathrm{ng} / \mu \mathrm{l}$ and sgRosa26-1 at $12.5 \mathrm{ng} / \mu \mathrm{l}, 80 \%$ of cultured embryos showed XbaI resistant PCR products, a percentage that was not further increased at higher concentrations (Fig. 1e, Additional file 1: Figure S1). XbaI resistant PCR products represented a minor fraction in most of the samples, indicating the preferential modification of the Rosa26 allele in a heterozygous and/or mosaic pattern, although $\sim 10 \%$ of the embryos showed processing of both alleles. We reasoned that conditions leading to Rosa26 deletions in the majority of embryos may also support knock-in events in at least a fraction of embryos, since HDR can occur in mammalian cells at $\sim 10 \%$ of nuclease induced DSBs [15].

\section{Knock-in of a conditional Cas9 transgene into Rosa26 of C57BL/6 zygotes}

To enable gene editing by CRISPR/Cas9 in vivo, we aimed for germ line integration of a conditional Cas9 transgene into the Rosa 26 locus of C57BL/6 mice such that the delivery of the large Cas 9 coding region into primary cells can be avoided. As a template for HDR, we constructed the targeting vector pRosa-Cas9, harboring an $11 \mathrm{~kb}$ insert flanked by standard Rosa26 homology regions, extending $1 \mathrm{~kb}$ upstream and $4 \mathrm{~kb}$ downstream from the XbaI site mentioned above (Fig. 2a). The vector's insert includes a CAG promoter region, a loxP-flanked transcriptional termination (Lox-Stop-Lox; LSL) element and the Cas9 coding region linked to an IRES-GFP reporter element. In addition, splice acceptor and polyA elements were placed upstream of the CAG promoter for the termination of the endogenous Rosa26 transcripts (Fig. 2a). From pronuclear microinjections and transfer of 207 C57BL/6 zygotes with pRosa-Cas9 DNA, sgRosa26-1 and Cas9-162A RNAs we obtained 38 live pups (Table 1). To verify the activity of Cas9 in microinjected zygotes, these 


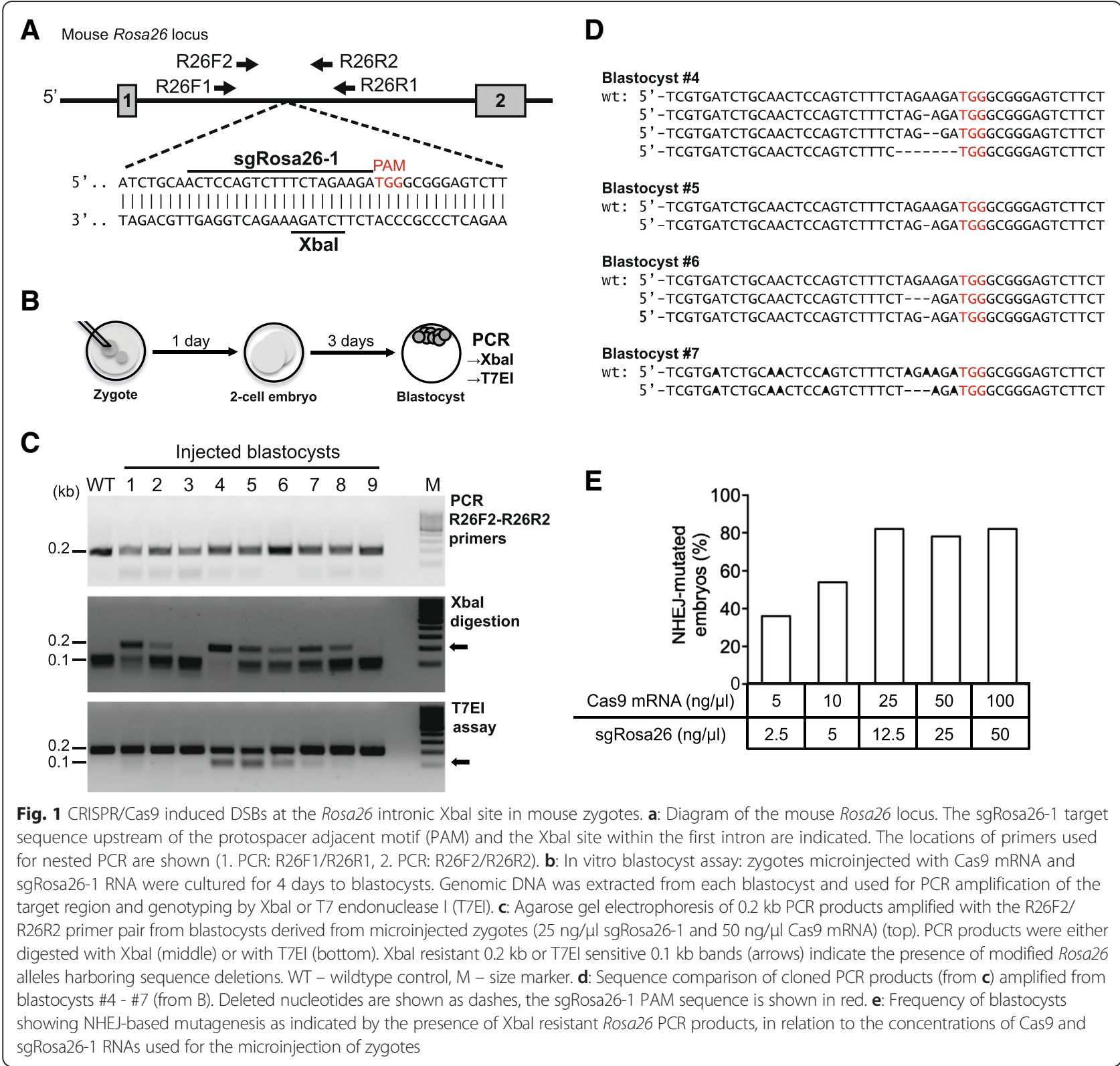

mice were first analyzed for the incidence of small deletions at the Rosa26 target site. PCR amplification of the target region on genomic DNA from ear biopsies using the primer pair R26F2/R2 and the XbaI digestion assay confirmed the presence of XbaI resistant, NHEJ processed Rosa26 alleles in 28 of 38 pups (74 \%) (Fig. 2b). Next, we used a Cas9-specific primer pair for PCR and identified six mice harboring the Cas9 transgene (Fig. 2c). These potential founder mutants were further analysed to discriminate knock-in alleles from random vector integrations. None of these mice showed knock-in to both Rosa26 alleles since additional wildtype or XbaI resistant PCR products were detected using the R26F2/SAR/ R2 or F2/R2 primer combinations (Fig. 2c). For the detection of correct, targeted integrations by PCR, we used the R26F3 primer, recognizing a genomic sequence outside of the upstream homology region of the targeting vector, together with the vector specific primer SAR. The predicted $1.38 \mathrm{~kb}$ PCR product could be amplified from five of the six Cas9 transgenic mice, indicating the correct configuration of the knock-in allele in founders \#18, \#20, \#35, \#36 and \#39 (Fig. 2d). Sequence analysis of these PCR products confirmed their identity as being derived from Rosa26 ${ }^{\text {LSL-Cas9 }}$ HDR alleles (Additional file 1: Figure S2). In 4 of 5 founders, Southern blot analysis of EcoRI digested tail DNA using a Rosa26-specific 5' -hybridization probe showed the predicted $6.0 \mathrm{~kb}$ band and thus correctly targeted alleles, whereas founder \#20 exhibited a 


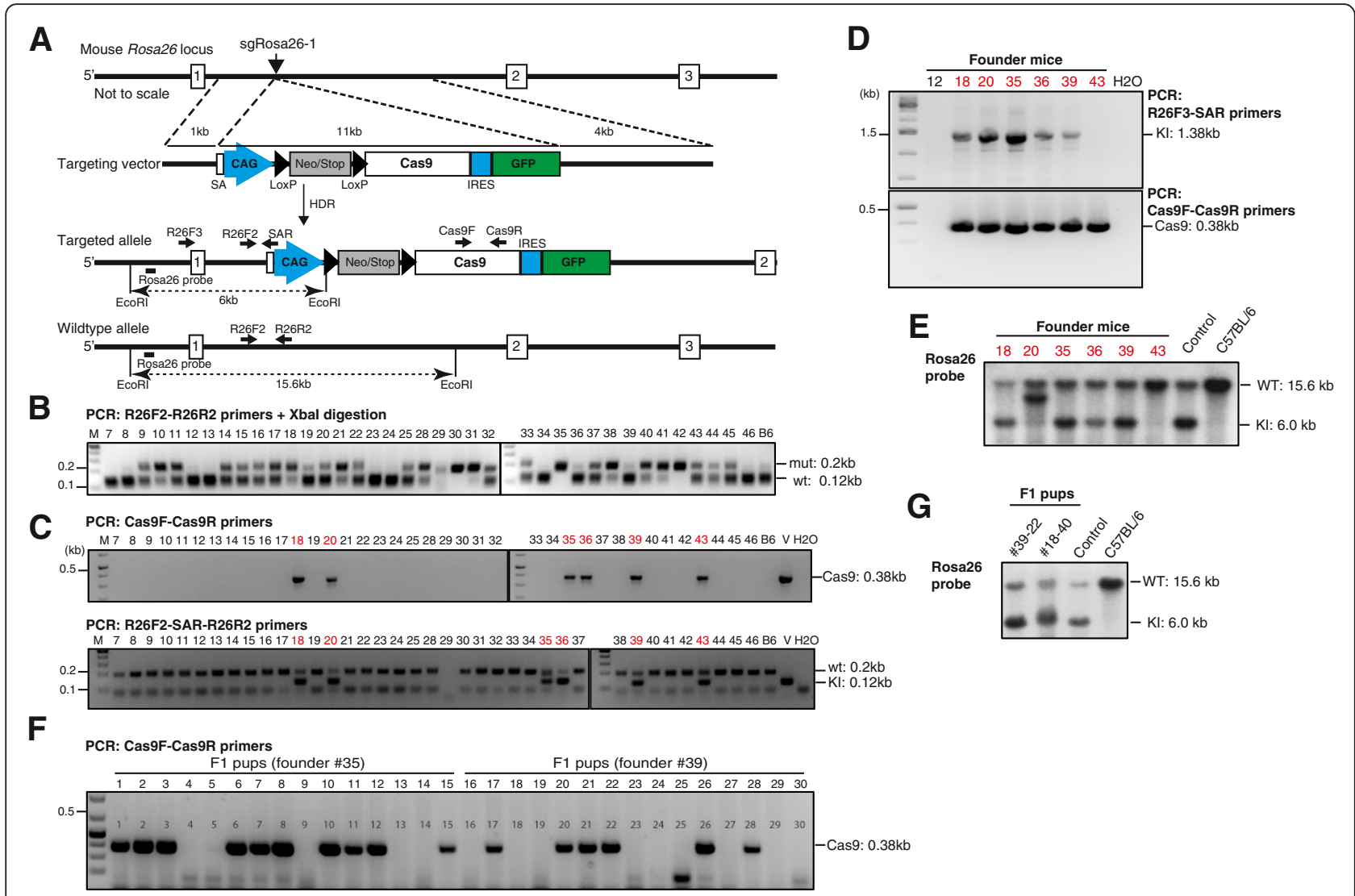

Fig. 2 Knock-in of a conditional Cas9 transgene into Rosa26 of C57BL/6 zygotes. a: Strategy for insertion of the CAG-loxPSTOPloxP-Cas9-IRES-EGFP cassette into the mouse Rosa26 locus. sgRosa26-1 and Cas9 introduce a double-strand break between $1 \mathrm{~kb}$ and $4 \mathrm{~kb}$ fragments used as homology arms in the targeting vector. Homology-directed repair (HDR) leads to the insertion of the cassette into the genome. The locations of PCR primers, restriction sites and the Rosa26 hybridisation probe in the targeted and wildtype alleles are indicated. $\mathbf{b}$ : Gel electrophoresis of Xbal digested Rosa26 PCR products (R26F2/R2 primers) amplified from pups (\#7-\#46) derived from microinjections of targeting vector, sgRosa26-1 and Cas9 RNAs. $0.2 \mathrm{~kb}$ bands of Xbal resistant products (mut) indicate sequence deletions, wildtype products (wt) are reduced to $0.1 \mathrm{~kb}$. M - size marker, B6 - C57BL/6 wildtype control. c: PCR detection of an internal segment of Cas9 in pups derived from microinjections using primers Cas9F/ Cas9R (top). Bottom: three primer PCR for the simultaneous detection of the Rosa26 target region (R26F2/R2 primers, $0.2 \mathrm{~kb}$ ) and of vector sequences (R26F2-SAR, $0.12 \mathrm{~kb}$ ), showing that all samples harbor at least one nonrecombined Rosa26 allele. $\mathrm{V}$ - vector positive control, $\mathrm{H}_{2} \mathrm{O}-$ negative control. d: Cas9-positive mice (from b) were further tested for correct knock-in (KI) into Rosa26 using a PCR reaction with a forward primer located outside of the $5^{\prime}$-homology region (R26F3) and a reverse primer located in transgene (SAR); the predicted band has a size of $1.38 \mathrm{~kb}$ (top). Bottom: DNA quality was controlled with a Cas9 internal PCR (Cas9F/R primers, $0.38 \mathrm{~kb}$ ). $\mathrm{H}_{2} \mathrm{O}$ - negative control. e: Southern blot analysis of EcoRI digested tail DNA from Cas9-positive mice (from b) using an external Rosa26-specific hybridization. Knock-in alleles are predicted to show a 6 kb band. Control - DNA from a Rosa26 knock-in mouse generated from ES cells, C57BL/6 - wildtype control. f: Genotyping PCR of 15 F1 pups derived from founder mutants \#35 or \#39 using the Cas9 internal primer pair Cas9F/R. g: Southern blot analysis of EcoRI digested tail DNA from two F1 pups using an external Rosa26-specific hybridization probe. Control - DNA from a Rosa26 knock-in mouse generated from ES cells, C57BL/6 - wildtype control

Table 1 Knock-in into the mouse Rosa26 locus using sgRNA and Cas9 mRNA

\begin{tabular}{lllllll}
\hline Donor vector & Concentration $(\mathrm{ng} / \mathrm{\mu l})$ & Injected zygotes & Transferred embryos & Live pups (\%) & Deletion alleles (\%) & Knock-in alleles (\%) \\
\hline Rosa26 & 10 & 105 & 60 & $7(12)$ & $3(43)$ & $0(0)$ \\
& 20 & 183 & 147 & $31(21)$ & $25(80)$ & $5(16)$ \\
Rosa26 & & 142 & 96 & $10(10)$ & ND & $2(20)$ \\
\hline
\end{tabular}


larger band, in addition to the $15.6 \mathrm{~kb}$ fragment from the Rosa26 wildtype locus (Fig. 2e). For germline transmission of the targeted alleles, founders \#18, 35, 36 and 39 were crossed to C57BL/6 wildtype mice and their offspring were genotyped using the Cas9 internal Cas9F/R primer pair. All founders transmitted the Rosa2 $6^{\text {LLL-Cas9 }}$ allele to about half of their offspring (Fig. 2f, Table 2). The Rosa26 loci of one pup each from founder \#18 (\#18-40) and \#39 (\#39-22) were further analyzed by Southern blotting of EcoRI digested genomic DNA using an external Rosa26 5' hybridization probe. Both pups showed the expected $6.0 \mathrm{~kb}$ band for the heterozygous Rosa $26^{\text {LSL-Cas9 }}$ allele, in addition to the $15.6 \mathrm{~kb}$ band derived from the Rosa26 wildtype locus (Fig. 2g).

Thus, using Cas9 and sgRosa26-1 RNAs, we achieved the targeted integration of an $11 \mathrm{~kb}$ conditional Cas9 transgene into the Rosa26 locus of C57BL/6 zygotes at a frequency of $13 \%$ and the Rosa $26^{\text {LSL-Cas9 }}$ founder mutants transmitted the targeted allele through their germ line.

\section{Cas9 is functional in B cells of Rosa26 $6^{\text {LSL-Cas9 }}$ mice}

To confirm the functionality of the Rosa $26^{\text {LSL-Cas9 }}$ allele, we isolated naive $B$ cells from spleens of three heterozygous $F_{1}$ mice by using $C D 43$ microbeads because the CD43 antigen is expressed on nearly all mouse leukocytes except for immature and resting mature B cells. The B cells were treated with cell permeable Tat-Cre recombinase for deletion of the loxP-flanked stop element, activated with LPS, inducing B cell proliferation and differentiation, for 2 days. The activated $\mathrm{B}$ cells were harvested and used for isolation of genomic DNA and cellular proteins (Fig. 3a). As shown by a three primer PCR for the detection of the recombined alleles, Tat-Cre removed the stop element with high efficiency (Fig. 3b) and sequence analysis of the PCR products confirmed the presence of a single loxP site between the CAG promoter and the Cas9 coding region (Fig. 3c). The expression of Cas9 protein from the activated Rosa $26^{\text {LSL-Cas9 }}$ allele was analyzed by Western blotting using lysates of Tat-Cre treated B cells and Cas9 or Flag-Tag specific antibodies. Both antibodies verified the expression of the $156 \mathrm{kD}$ Cas9 protein in Tat-Cre treated B cells from three heterozygous Rosa26 ${ }^{\text {LSL-Cas9 }}$ mice (Fig. 3d).
The nuclease activity of the expressed Cas9 protein was confirmed by the transduction of Tat-Cre treated, LPS activated B cells with retroviral particles expressing sgRosa26-1, a puromycin resistance and a BFP gene (Fig. 4a). The transduced B cells of F1 Rosa26 ${ }^{\text {LSL-Cas9 }}$ heterozygous pups from three different founders (\#18, \#35 and \#39) were selected with puromycin for three days, leading to an enrichment of $\mathrm{BFP}^{+}$transduced cells to $90 \%$ (Fig. 4b). We then isolated genomic DNA from FACS sorted $\mathrm{BFP}^{+}$cells from the experimental and control cultures and performed PCR amplification of the sgRosa26-1 target region, followed by Xbal digestion and T7EI assays. In both groups, we found high levels of XbaI resistant and T7E sensitive Rosa26 PCR products (Fig. 4c), indicating sufficient Cas9 expression from the conditional Rosa $26^{\text {LSL-Cas9 }}$ allele to achieve targeted mutagenesis.

These results verified the expression of functional Cas9 nuclease in Rosa26 ${ }^{\text {LSL-Cas9 }} \mathrm{F}_{1}$ offspring upon Cremediated activation of the transgene. Since the targeted allele was introduced into C57BL/6 zygotes, the Rosa26 ${ }^{\text {LSL-Cas9 }}$ mouse line allows Cas9-mediated gene editing in vivo and in primary cells of C57BL/6 mice. The Rosa $26^{\text {LSL-Cas9 }}$ line will be distributed through the Jackson laboratory (www.jax.org).

\section{Analysis of off-target activity}

Genomic sequences showing high similarity to the sgRosa26-1 target-sequence may lead to unintended gene editing at such off-target sites. To determine the importance of off-target modification in our system, we predicted the off-target sites of sgRosa26-1 in the mouse genome based on sequence similarity to its targetsequence and selected the three sites with the highest risk of being edited (Fig. 5a). We then amplified and sequenced these loci from six heterozygous Rosa26 ${ }^{\text {LSL-Cas9 }}$ $F_{1}$-mice (derived from the founder mutants \#18, \#35 and $\# 39$ ) and from the six Cas9 positive mice of the founder generation (\#18, \#20, \#35, \#36, \#39, \#43). We did not detect any genetic modification in all of the analysed offtarget loci, since solely wildtype, but no mixed sequence reads were obtained (Fig. 5b-d, Additional file 1: Figure S3). Although we cannot rule out off-target effects in other loci, these results suggest that in the present setting, off-target effects are not dominant.

Table 2 Germline transmission of Rosa26 $6^{\text {LSL-Cas9 }}$ alleles

\begin{tabular}{lllll}
\hline Rosa26 & \# pups & Male & Female & Rosa26 \\
\hline \#18 (female) & 9 & 7 & 2 & $5(55)$ \\
\#35 (male & 15 & 8 & 7 & positive $(\%)$ \\
\#36 (male) & 8 & 4 & 4 & $10(66)$ \\
\#39 (male) & 17 & 8 & 9 & $5(47)$ \\
\hline
\end{tabular}




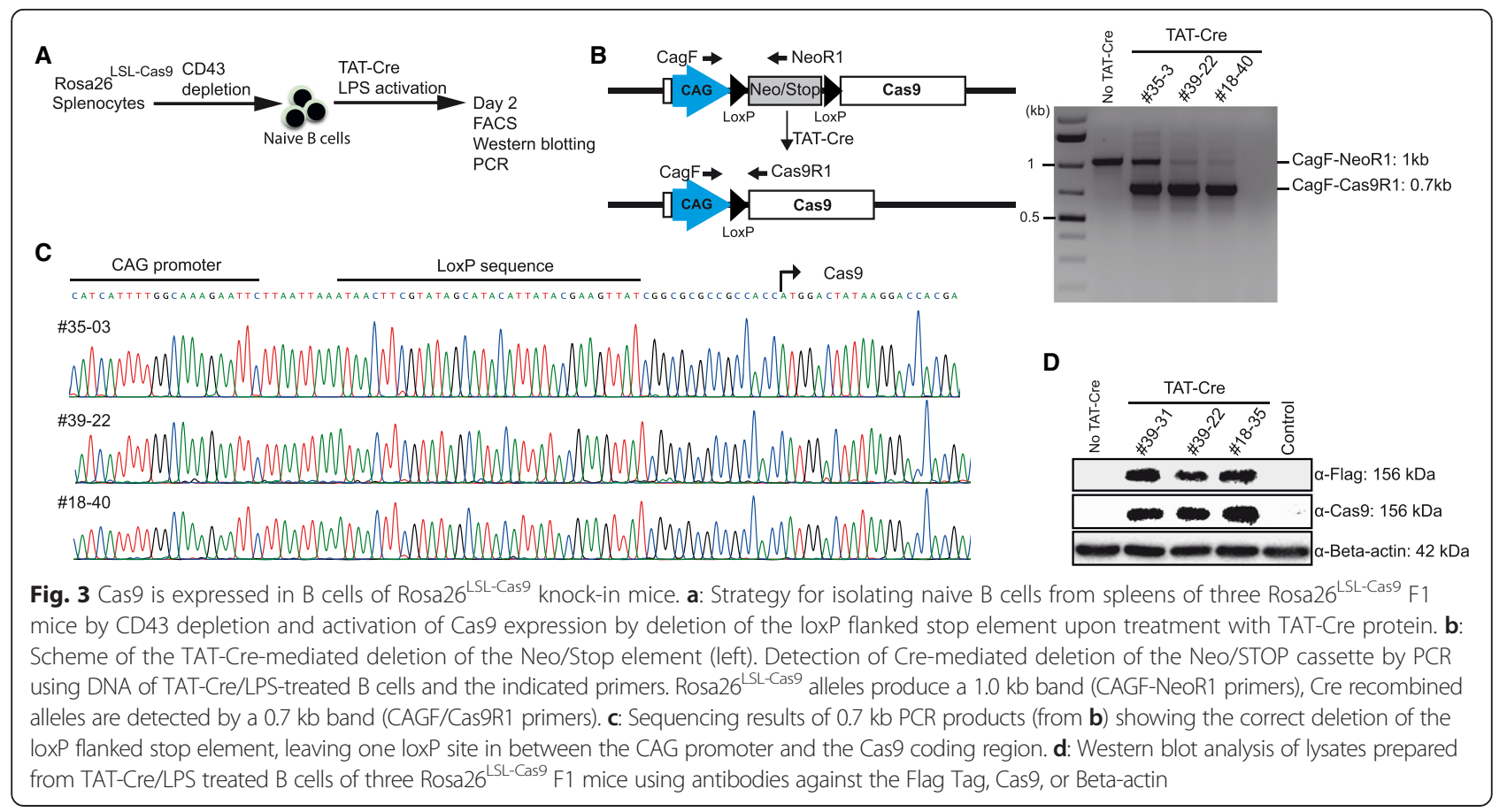

\section{Knock-in of a conditional Galectin-1-E2A-PD-L1 transgene into Rosa26 of C57BL/6 zygotes}

Evidence that the Rosa26 targeting strategy outlined above can be extended to other transgenes was obtained in experiments aiming at the generation of signal-on alleles encoding the immunomodulatory proteins Galectin1 (Lgals1) and PD-L1 (Cd274), linked by a self-cleaving E2A peptide. For this purpose we constructed a Rosa26 targeting vector by inserting the $8 \mathrm{~kb}$ transgene into a Gateway cloning destination vector [16], harboring $\lambda$ phage attR sites in between the loxP flanked stop element and an IRES-GFP reporter gene (Fig. 6a). For knock-in into the Rosa26 locus the Lgals1-E2A-Cd274 targeting vector was microinjected together with sgRosa26-1 $(12.5 \mathrm{ng} / \mu \mathrm{l})$ and Cas9-162A (25 ng/ $\mu \mathrm{l}) \mathrm{RNAs}$ into the pronuclei of $142 \mathrm{C} 57 \mathrm{BL} / 6$ zygotes. To control for embryo viability, the injected zygotes were cultured overnight to the 2-cell stage. We recovered 96 such embryos (68\%) that, upon transfer into foster mothers, resulted in the birth of 10 pups (Table 1). Genomic DNA from ear biopsies was first used for the detection of vector integrations by PCR amplification of an internal segment of the stop element using the NeoF/R primer pair (Fig. $6 \mathrm{~b}$ ). Four pups showed the predicted 324 bp band and were further genotyped using the R26F3/SAR primer pair, enabling the detection of Rosa26 HDR alleles by a forward primer (F3) outside of the vector's upstream homology region and a vectorspecific reverse primer (SAR). The predicted $1.38 \mathrm{~kb}$ PCR product could be amplified from 2 pups (\#90 and \#95), confirming the presence of Rosa26 knock-in alleles
(Fig. 6b). The presence of the protein coding segment was further confirmed using an Lgals1 and Cd274 specific primer pair. In addition, we verified the correct integration of the targeting vector in both founders by Southern blot analysis of EcoRI digested tail DNA using an external Rosa26 5' hybridization probe. Both founders showed the $6.0 \mathrm{~kb}$ band predicted for the Rosa2 $6^{\text {Lgals-Cd274 }}$ allele, in addition to the $15.6 \mathrm{~kb}$ band derived from the Rosa26 wildtype locus (Fig. 6b). The two founder mutants are presently bred for germline transmission of the targeted Rosa26 $6^{\text {LSL-Lgals/Cd274 }}$ allele.

In conclusion, the present direct targeting approach using CRISPR/Cas9 in zygotes allows the rapid generation of new Rosa26 knock-in mouse lines on the C57BL/6 inbred background. Since 10-17\% of transferred embryos developed into live pups and $10-20 \%$ of them were correctly targeted mutants, the establishment of a new knock-in line requires the microinjection and transfer of no more than 100-200 zygotes.

\section{Resources for Rosa26 targeting in zygotes}

To facilitate the construction of new conditional Rosa26 targeting vectors, we provide targeting vectors harboring pairs of $\lambda$-attR sites for the insertion of coding regions in between a loxP flanked stop element and an IRES-GFP or -BFP reporter by Gateway cloning (Fig. 7). Each of these versions either contains the CAG promoter for transgene expression or an acceptor element for splicing to the endogenous Rosa26 transcript. In addition, we provide conditional targeting vectors enabling the 
A

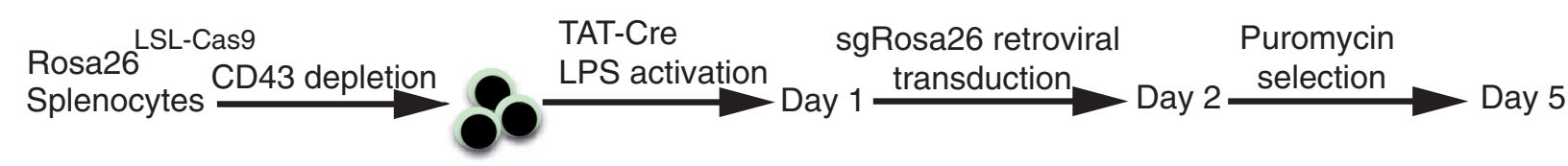

Naive B cells

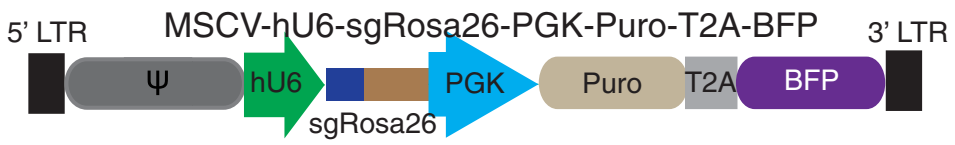

B

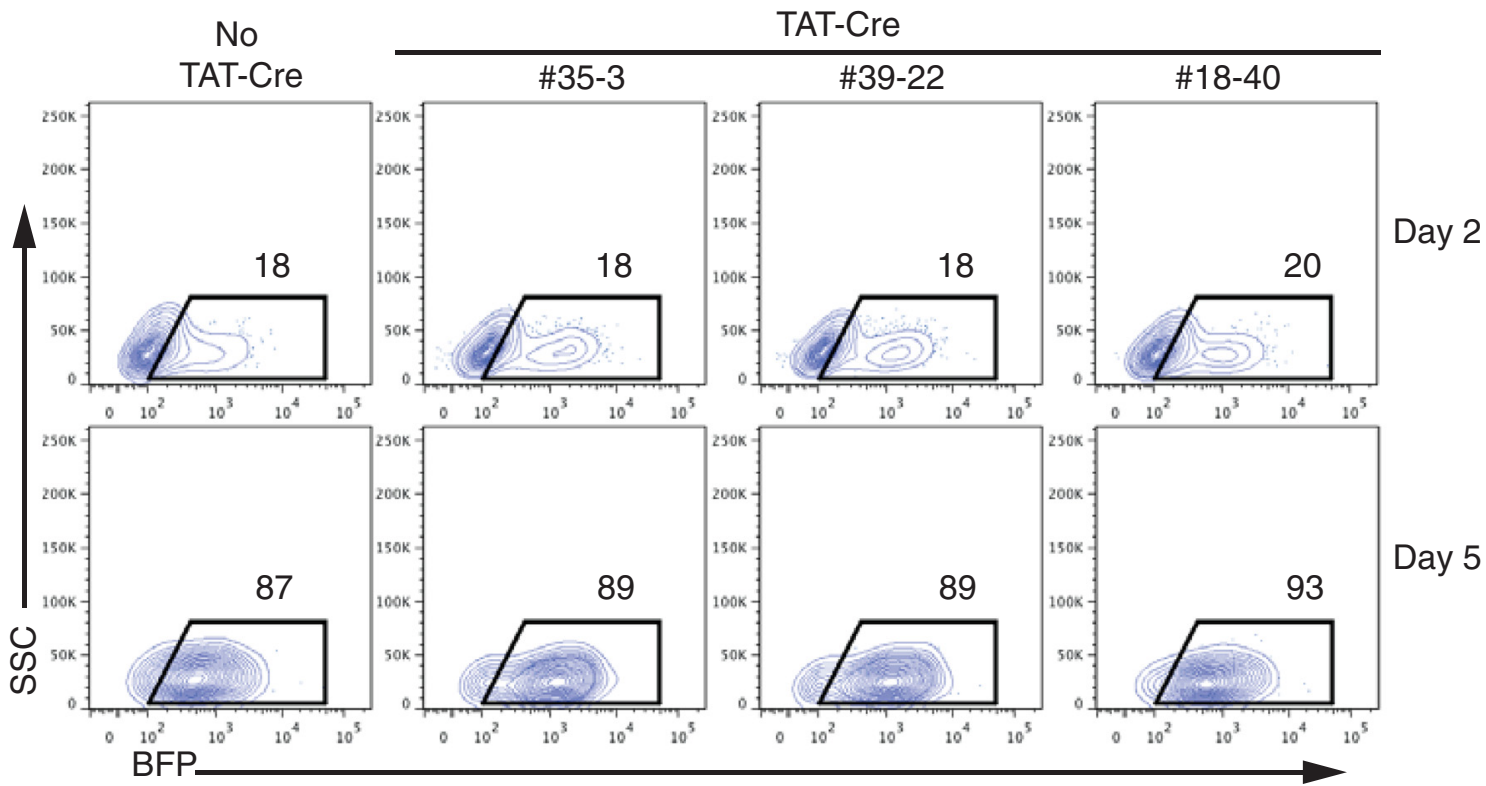

C

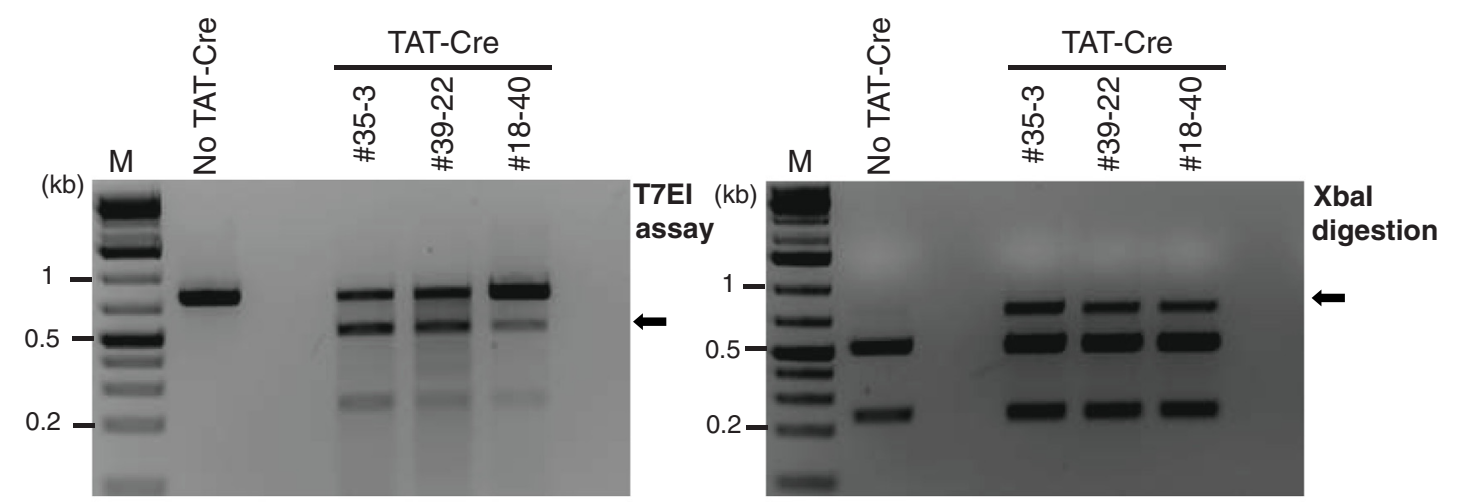

Fig. 4 Cas9 is functional in B cells of Rosa26 $6^{\text {LSL-Cas9 }}$ knock-in mice. a: Scheme of genome editing in primary mouse B cells using CRISPR/Cas9. Naive B cells from spleens of three individual heterozygous Rosa26 $6^{\text {LSL-Cas9 }} \mathrm{F} 1$ mice were isolated using CD43 depletion, treated with TAT-Cre and stimulated with LPS for $24 \mathrm{~h}$. TAT-Cre/LPS treated B cells were transduced with retroviral particles co-expressing sgRosa26-1 and BFP to target the Rosa26 locus. One day later, the transduced B cells were selected with puromycin until day 5. b: FACS analysis of B cells (from a) before (day 2) and after puromycin selection (day 5). The gate indicates the fraction (percentage) of successfully transduced BFP ${ }^{+}$cells. c: Gel electrophoresis of T7El or Xbal digested PCR products (R26T7F/R26T7R primers) amplified from DNA of FACS sorted BFP ${ }^{+}$cells (from $\mathbf{b}$ ), indicating sequence deletions by the presence of T7El sensitive or Xbal resistant bands (arrows) 
A

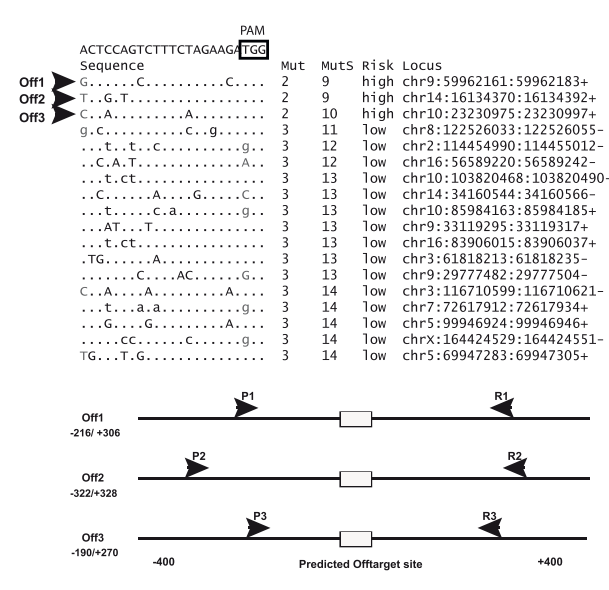

B

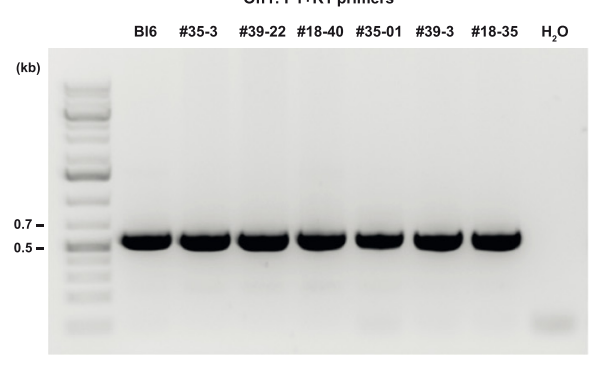

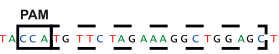

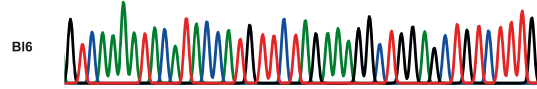

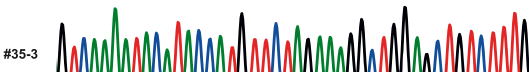

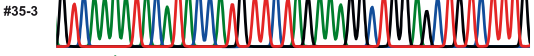

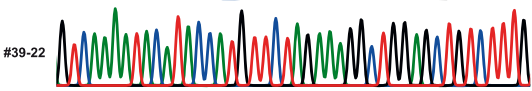
*18-40 A

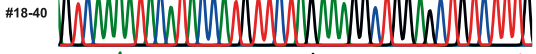

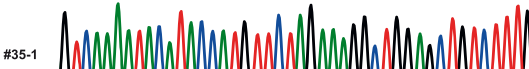

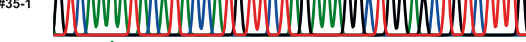

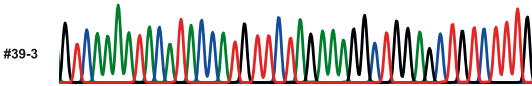

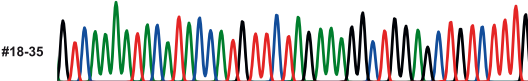

C

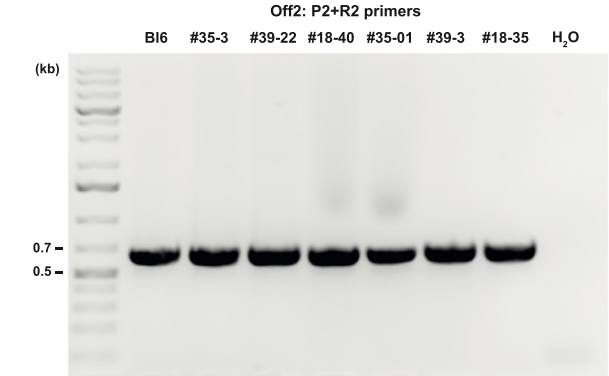

C

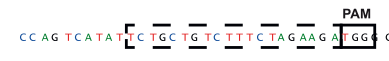

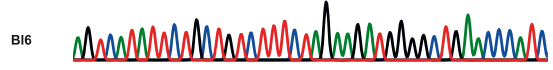

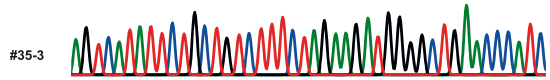

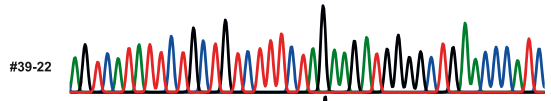

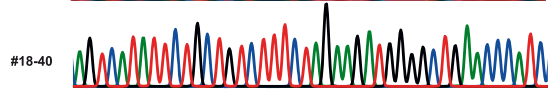

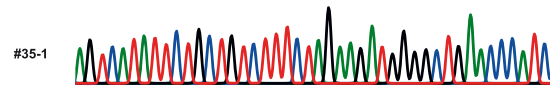

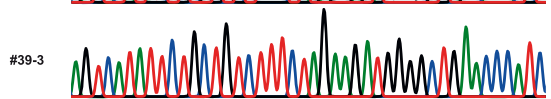

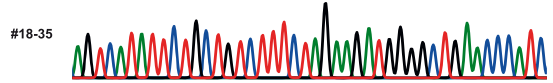

D

(kb) B16 \#35-3 \#39-22 \#18-40 \#35-01 \#39-3 \#18-35 $\mathrm{H}_{2} \mathrm{O}$

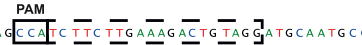

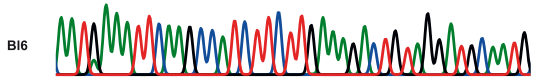

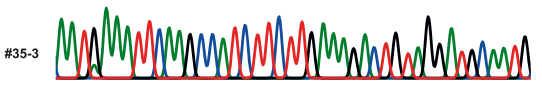

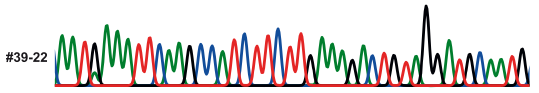

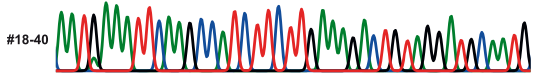

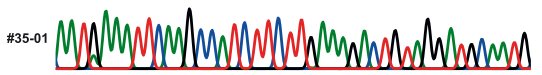

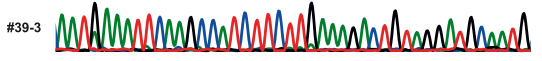

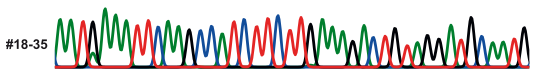


(See figure on previous page.)

Fig. 5 Analysis of off-target activity. a: The top 18 predicted off-target sites of the Rosa26-1 target sequence sorted according to sequence divergence (upper panel) and the PCR scheme for the analysis of the top 3 off-targets (lower panel). Negligible mismatches are shown in grey. $\mathbf{b}$ : PCR amplification of the off-target site 1 (Off1) from two F1 pups each derived from the mutant founders \#18, \#35 or \#39 (upper panel) and sequencing results of the respective bands (lower panel, Bl6-C57BI/6 wildtype control). c: PCR amplification of the off-target site 2 (Off2) from two F1 pups each derived from the mutant founders \#18, \#35 or \#39 (upper panel) and sequencing results of the respective bands (lower panel, Bl6-C57BI/6 wildtype control). d: PCR amplification of the off-target site 3 (Off3) from two F1 pups each derived from the mutant founders \#18, \#35 or \#39 (upper panel) and sequencing results of the respective bands (lower panel, Bl6-C57BI/6 wildtype control)

standard cloning of new inserts into an AscI site located upstream of an IRES-GFP reporter or into the AsiSI or MluI site of a reporter-free plasmid (Fig. 7). For the preparation of sgRosa26-1 and Cas9-162A RNAs for microinjection we provide plasmids pBS-U6-sgRosa26-1 and pCAG-Cas9-162A. All plasmids will be distributed via the Addgene repository (www.addgene.org) and protocols for RNA production and the PCR-based detection of modified Rosa26 alleles and off-target analysis are included in the Additional file 2.

In addition to the standard conditions with sgRosa261 and Cas9-162A RNAs, as used for the generation of Rosa $26^{\text {LSL-Cas9 }}$ and Rosa26 $6^{\text {LSL-Lgals/Cd274 }}$ mice, we explored whether the frequency of knock-in events can be further increased by the co-injection of recombinant Cas9 protein. For these test experiments we cultured microinjected zygotes to the blastocyst stage, extracted genomic DNA and determined the frequency of Rosa26 knock-in and deletion events by PCR. For the knock-in into Rosa26, we used a Venus targeting vector harboring the $1 \mathrm{~kb}$ standard $5^{\prime}$-homology region and a $3^{\prime}$-homology region shortened from $4 \mathrm{~kb}$ to $0.8 \mathrm{~kb}$ (Fig. 8a) to facilitate the detection of recombined alleles by PCR using the vector-specific primer VenusF and the external downstream R26R3 primer (Table 3). We analyzed two groups of 12 blastocysts each that were derived from the microinjection of zygotes with aliquots of an identical preparation of sgRosa26-1 RNA, Cas9-162A mRNA and pRosa26-Venus DNA, except that one sample was supplemented with Cas 9 protein $(30 \mathrm{ng} / \mu \mathrm{l})$ immediately before injection. As shown in Fig. 8b, we found two embryos (17 \%) positive for the 1.38 kb knock-in PCR product in the group microinjected with the RNA/DNA preparation alone, comparable to our previous results. In the group microinjected with additional Cas9 protein 6 of 12 embryos (50\%) were positive for the knock-in PCR product. Although the small sample size prevents statistical evaluation, it is possible that the combined use
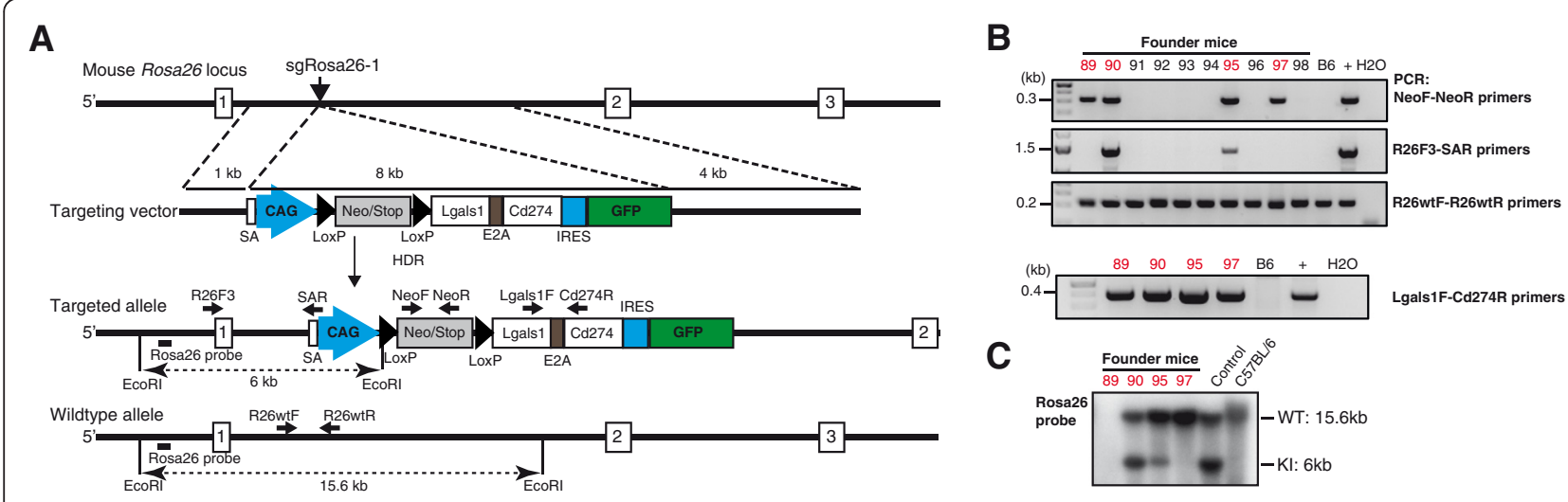

Fig. 6 Knock-in of a conditional Galectin-1-E2A-PDL1 transgene into Rosa26 of C57BL/6 zygotes. a: Strategy for insertion of the CAG-loxPSTOPloxPLgals1-E2A-Cd274-IRES-EGFP cassette into the mouse Rosa26 locus. sgRosa26-1 and Cas9 introduce a double-strand break between $1 \mathrm{~kb}$ and $4 \mathrm{~kb}$ fragments used as homology arms in the targeting vector. Homology-directed repair (HDR) leads to the insertion of the cassette into the genome. The locations of PCR primers, restriction sites and the Rosa26 hybridisation probe in the targeted and wildtype alleles are indicated. b: Gel electrophoresis of PCR reactions from genomic DNA of ten pups derived from microinjections using primers NeoF/R for detection of an internal vector segment (stop element, top). Second panel: Mouse DNAs were further tested for correct knock-in (KI) into Rosa26 using a PCR with a forward primer located outside of the 5'-homology region (R26F3) and a reverse primer located in transgene (SAR); the predicted band has a size of $1.38 \mathrm{~kb}$. Third panel: DNA quality was controlled with a Rosa26-specific PCR (R26wtF/R primers, $0.2 \mathrm{~kb}$ ). Lower panel: PCR detection of the Galectin-1-E2A-PDL1 transgene using Lgals1F (forward) and Cd274R (reverse) primers. +: Positive control DNA from a Rosa26 knock-in mouse generated from ES cells; $\mathrm{H}_{2} \mathrm{O}$ : negative control. c: Southern blot analysis of EcoRl digested tail DNA from vector-positive mice (from B) using an external Rosa26-specific hybridization probe. Knock-in alleles are predicted to show a $6 \mathrm{~kb}$ EcoRl band; for sample \#89 the tail biopsy yielded insufficient gDNA. Control - DNA from a Rosa26 knock-in mouse generated from ES cells, C57BL/6 - wildtype control 


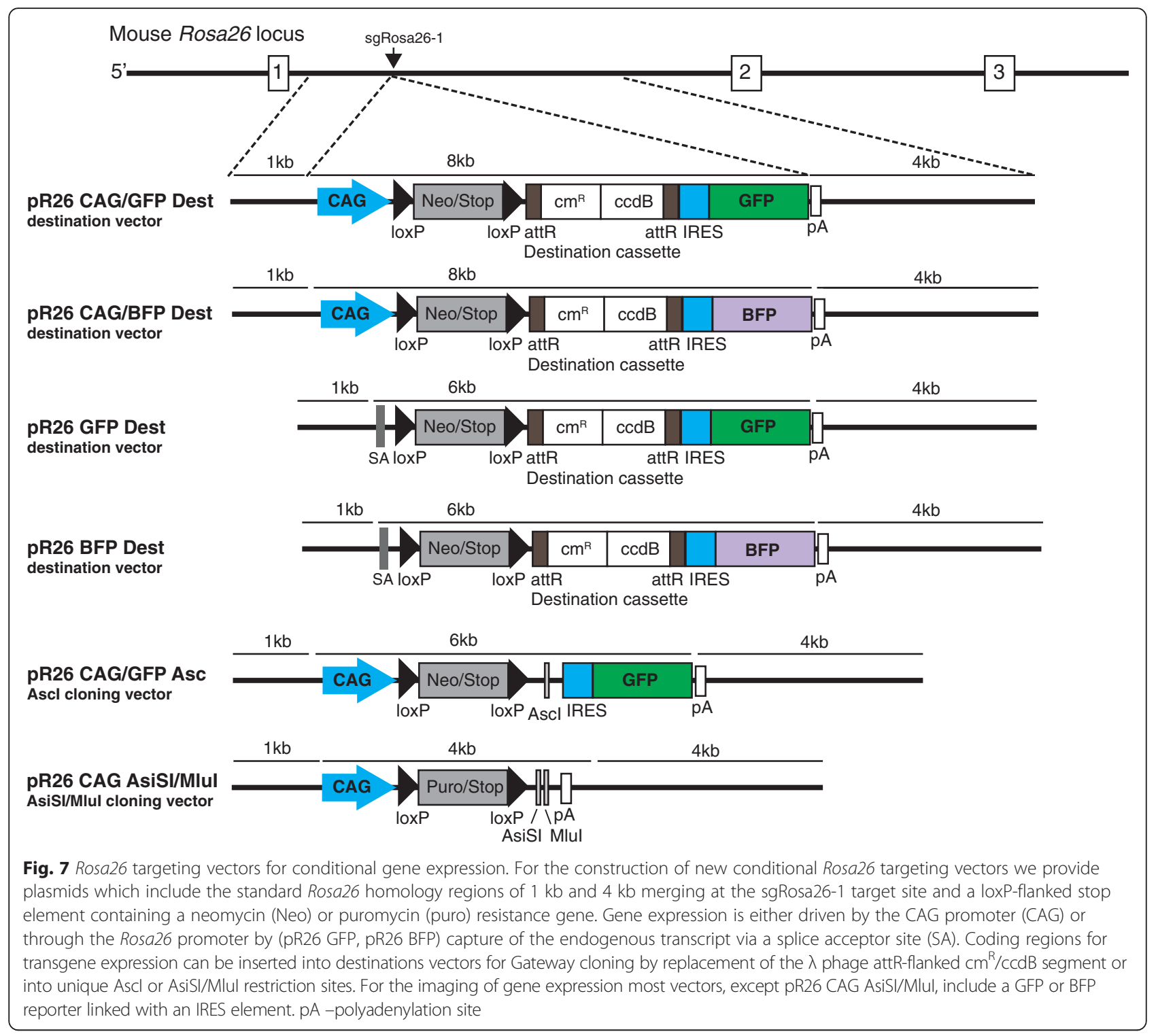

of Cas9 mRNA and protein leads to increased Cas9 cleavage and improved HDR. The frequency of small deletions at the Rosa26 target site, as assessed by XbaI digestion of PCR products, was also clearly elevated in embryos co-injected with Cas9 protein (Fig. 6b).

\section{Discussion}

Efficient gene editing in mouse zygotes using the CRISPR/Cas9 system has been mainly reported for the generation of knockout alleles by small sequence deletions in genetically hybrid embryos. However, many research applications require precisely targeted mutations on the C57BL/6 inbred background. Specifically, targeted insertions into the Rosa26 locus are frequently used for the constitutive or conditional expression of transgenes in a standardized single copy configuration. Here we established a CRISPR/Cas9 based approach for generating Rosa26 knock-in alleles in C57BL/6 zygotes. We found that transgene inserts of 8 or $11 \mathrm{~kb}$ were targeted to Rosa26 in 10-20\% of the mice derived from microinjections of 100-200 C57BL/6 zygotes. For the detection of potential off-target modifications by Cas9, we tested six Rosa $26^{\text {LSL-Cas9 }} F_{1}$ pups each at three predicted off-target sites. Since these sites showed no modifications, we conclude that sgRosa26-1 does not lead to obvious, frequent off-target processing.

To facilitate the future production of Rosa 26 knock-in mouse lines, we provide various targeting constructs for the insertion of new transgenes using Gateway or restriction site cloning. Furthermore, we found a higher 


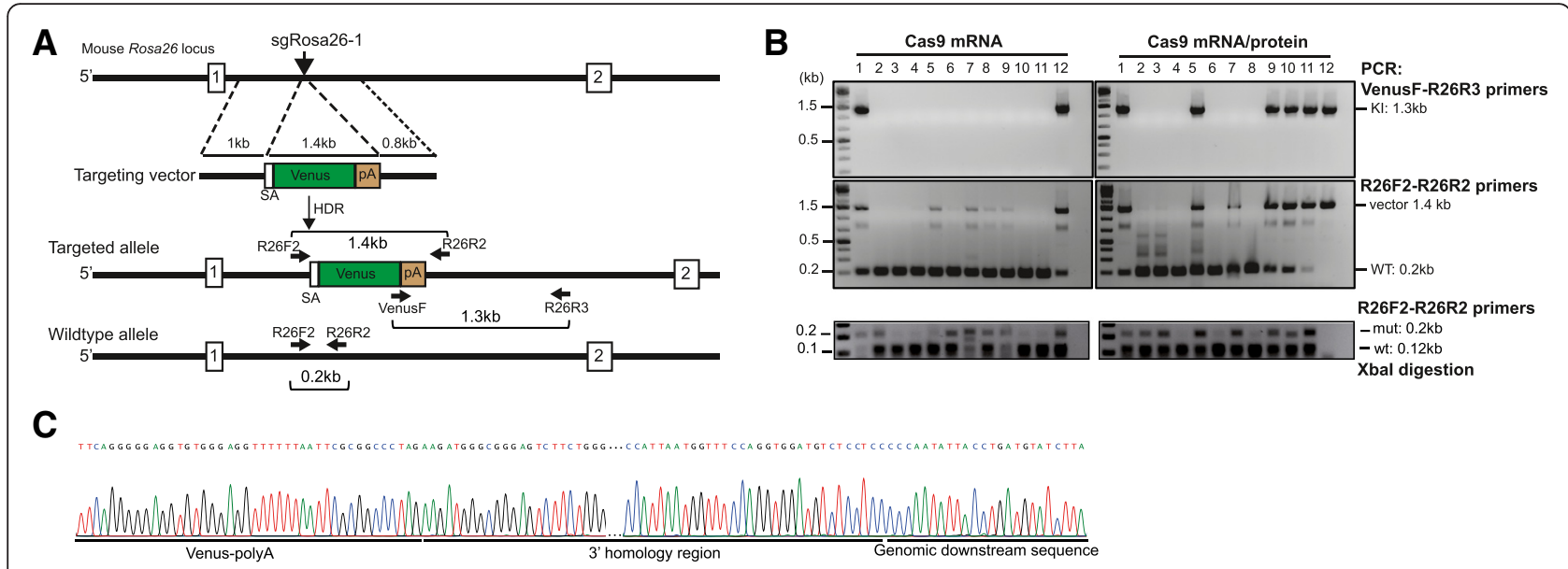

Fig. 8 Coinjection of Cas9 mRNA and Cas9 protein into zygotes. a Strategy for insertion of a Venus reporter into the mouse Rosa26 locus. sgRosa26-1 and Cas9 introduce a double-strand break between $1 \mathrm{~kb}$ and $0.8 \mathrm{~kb}$ fragments used as homology arms in the pR26-Venus targeting vector. The locations of PCR primers in the targeted and wildtype Rosa26 alleles are indicated. SA- splice acceptor, pA polyadenylation site. $\mathbf{b}$ Mouse zygotes were microinjected with pR26-Venus, sgRosa26-1 and Cas9 mRNA or Cas9 mRNA and protein. The embryos were cultured for 4 days and genomic DNA was isolated from 12 blastocysts each, for PCR-based detection of HDR or deletion events. Top panel: gel electrophoresis of PCR products. Targeted alleles (KI) are detected by amplification of a $1.3 \mathrm{~kb}$ genomic segment using the vector-specific primer VenusF and the R26R3 primer, located downstream of the vector homology region. The presence of integrated or nonintegrated vector DNA was tested using the R26F2/R2 primer pair, amplifying a $1.4 \mathrm{~kb}$ vector segment as well as $0.2 \mathrm{~kb}$ of the Rosa26 target region (middle panel). Lower panel: Rosa26 alleles with sequence deletions were detected by $0.2 \mathrm{~kb}$ of the target region (R26F2/R26R2 primers), followed by Xbal digestion and gel separation. Xbal resistant PCR products indicate the presence of sequence deletions (mut, $0.2 \mathrm{~kb}$ ) whereas wildtype products are reduced to $0.12 \mathrm{~kb}$ fragments (wt). c Sequencing of PCR products amplified with primers VenusF and R26R3 (from B, top) showed the predicted recombination between the targeting vectors homology region and adjacent downstream genomic sequence

proportion of Rosa26 knock-in alleles in a small group of embryos microinjected with Cas9 mRNA and additional Cas9 protein. Although this result is out of reliable statistical evaluation, it is possible that the microinjection of Cas9 protein and sgRNA stimulates DSB formation and HDR early on, complementing and preceding the translation of Cas9 mRNA that supports sustained nuclease activity over time. Thus, provided that live mutants will be obtained at similar rates, the combined supply of Cas9 mRNA and protein could further streamline the production of Rosa26 and other knock-in mutants, provided that future experiments will confirm our initial findings. In addition, the suppression of NHEJ repair by inhibition of DNA Ligase IV $[13,17]$ may provide another option to increase the yield of Rosa26 HDR alleles in zygotes. Since we also successfully used a shortened, $0.8 \mathrm{~kb} \mathrm{3'}$ '-homology region for the knock-in of Venus into Rosa26, it will be interesting to further investigate which lengths of homology regions, in relation to the size of transgene inserts, are required for optimal HDR efficiency.

Previous studies on the direct targeting of Rosa26 used pairs of zinc-finger nucleases [7] or TALEN [18] in zygotes and achieved HDR frequencies of 1.7-4.5 \% (ZFN) or $5.8-11.7 \%$ (TALEN), respectively, for the insertion of reporter genes. Intrinsic limitations for DSB induction by these earlier nuclease designs may be posed by the requirement for binding of two protein molecules to the target DNA and for the dimerization of their nuclease domains. Since for Cas9, DSBs are formed by only one single protein/RNA complex we reason that higher levels of mutagenesis can be achieved by the delivery of preformed Cas9/sgRNA into zygotes. As an alternative to sequence-specific nucleases, Cre/loxP mediated recombination has been used in zygotes for vector integrations by recombinase-mediated cassette exchange (RMCE) into a modified Rosa26 allele at efficiencies of 4.4-25\% $[19,20]$. However, the RMCE approach is incompatible with the use of Cre/loxP dependent constructs and requires the maintenance of a breeding colony of RMCE acceptor mice for embryo production.

Using CRISPR/Cas9 we generated a new conditional Cas9 mouse line for in vivo and ex vivo gene editing in the C57BL/6 inbred background. A similar strain was previously generated by gene targeting in 129-derived R1 ES cells [21]. A minimum of 10 backcross cycles with C57BL/6 mice will be required for the establishment of a congenic Cas9 strain, precluding prompt phenotypic studies that require this widely used inbred background. Since Rosa26 has been also validated as a 'safe harbor' integration site in rats and pigs using ES cells [22] or nuclear transfer [23], CRISPR/Cas9-based Rosa26 knock-in in zygotes could also be of use in these species. Finally, 
efficient targeting of the Rosa26 locus in the C57BL/6 background allows the direct targeting of complex experimental compound mutants and thus bypassing timeconsuming breeding strategies.

\section{Conclusions}

Rosa26 is frequently used as standardized insertion site for single transgene copies via gene targeting in ES cells, an approach that requires laborious handling of cell cultures and 9-12 months' time until the identification of positive $F_{1}$ pups. Here we applied CRISPR/Cas9-assisted mutagenesis for the single step insertion of conditional transgenes into Rosa 26 of C57BL/6 zygotes. Using modified Cas 9 mRNA and sgRNA targeting the intronic XbaI site of Rosa26, we achieved the knock-in of 8-11 kb

Table 3 PCR primers used in this study

\begin{tabular}{|c|c|c|}
\hline PCR type & Primer & Sequence $\left(5^{\prime}>3^{\prime}\right)$ \\
\hline \multirow{4}{*}{$\begin{array}{l}\text { Nested PCR for } \\
\text { Rosa26 locus }\end{array}$} & R26F1 & CCAAAGTCGCTCTGAGTTGTTATCAGT \\
\hline & R26R1 & GGAGCGGGAGAAATGGATATGAAG \\
\hline & $\mathrm{R} 26 \mathrm{~F} 2$ & GCCTCCTGGCTTCTGAGGACCG \\
\hline & R26R2 & TCTGTGGGAAGTCTTGTCCCTCC \\
\hline \multirow[t]{2}{*}{ Cas9 transgene } & Cas9F & GGCATCCTGCAGACAGTGAAGGTGG \\
\hline & Cas9R & CGGTTCTTGTCGCTTCTGGTCAGCA \\
\hline \multicolumn{3}{|l|}{ Homozygous } \\
\hline \multirow{3}{*}{$\begin{array}{l}\text { Heterozygous for } \\
\text { R26-Cas9 allele }\end{array}$} & $\mathrm{R} 26 \mathrm{~F} 2$ & GCCTCCTGGCTTCTGAGGACCG \\
\hline & R26R2 & TCTGTGGGAAGTCTTGTCCCTCC \\
\hline & SAR & CCTGGACTACTGCGCCCTACAGA \\
\hline \multicolumn{3}{|l|}{ Long PCR for correct } \\
\hline \multirow[t]{2}{*}{ Integration } & $\mathrm{R} 26 \mathrm{~F} 3$ & CTGCCCGAGCGGAAACGCCACTGAC \\
\hline & SAR & CCTGGACTACTGCGCCCTACAGA \\
\hline \multirow[t]{2}{*}{ Neo transgene } & $\mathrm{NeoF}$ & GCTAACCATGTTCATGCCTTC \\
\hline & NeoR & CGTTGGCTACCCGTGATATT \\
\hline \multirow{2}{*}{$\begin{array}{l}\text { Loading PCR for } \\
\text { Rosa26 locus }\end{array}$} & R26wtF & GGAGTGTTGCAATACCTITCTGGGAGTTC \\
\hline & R26wtR & TGTCCCTCCAATTITACACCTGTTCAATTC \\
\hline \multirow{2}{*}{$\begin{array}{l}\text { Lgals-Cd274 } \\
\text { transgene }\end{array}$} & Lgals1F & CAAGATTAAGTGCGTGGCC \\
\hline & $\mathrm{Cd} 274 \mathrm{R}$ & CATTTCCCTTCAAAAGCTGGTC \\
\hline \multirow{2}{*}{$\begin{array}{l}\text { Venus correct } \\
\text { integration }\end{array}$} & VenusF & GGCCTCTCGAGCCTCTAGAACTATAGTG \\
\hline & R26R3 & CAAGCTCACAAGACCTTAGGTCAGGA \\
\hline \multirow{3}{*}{$\begin{array}{l}\text { LoxP-flanked Stop } \\
\text { cassette deletion }\end{array}$} & CagF & CAAGCTCACAAGACCTTAGGTCAGGA \\
\hline & NeoR1 & ATGGGATCGGCCATTGAACAAGATGG \\
\hline & Cas9R1 & CGGCCTTGTCGGTGCTGTCCACCAG \\
\hline \multirow{2}{*}{$\begin{array}{l}\text { PCR Rosa26 for T7EI } \\
\text { and RLFP }\end{array}$} & R26T7F & CGTGCAAGTTGAGTCCATCCGCC \\
\hline & R26T7R & ACTCCGAGGCGGATCACAAGCA \\
\hline
\end{tabular}

inserts in $10-20 \%$ of pups derived from microinjections of C57BL/6 embryos. Upon the combined microinjection of Cas9 mRNA and Cas9 protein we found knock-in alleles in $50 \%$ of cultured blastocysts. For modifying gene function by CRISPR/Cas9 in somatic tissues of $\mathrm{C} 57 \mathrm{BL} / 6$ mice, to avoid the vectormediated delivery of the large Cas 9 transgene into primary cells, we generated Rosa26 knock-in mice for the Cre/loxP-dependent expression of Cas9. Taken together, our protocols and resources support the fast and direct generation of new Rosa26 knock-in alleles and of Cas9-mediated in vivo gene editing in the widely used C57BL/6 inbred strain.

\section{Methods}

\section{Cloning of targeting vectors}

Targeting vectors were cloned by modifying a published Rosa26 targeting vector containing a loxP-flanked STOP cassette and an IRES-GFP reporter [24]. A CAG promoter, preceded by two copies of the bovine growth hormone gene poly(A)-addition signal, was inserted upstream of the STOP cassette using a PacI site as previously published [25]. A diphtheria toxin gene downstream of the 3' homology arm was removed using AgeI and AsisI restriction sites. cDNA coding for Cas9 was amplified from plasmid pX330 (Addgene \#42230) and was inserted into the targeting vector using an AscI restriction site. Lgals1 cDNA was amplified from sequence NM_008495.2, Cd274 cDNA was amplified from sequence NM_021893.3 (both from plasmids provided by the DNA Resource Core at Harvard Medical School) and the published sequence coding for E2A was purchased as DNA oligonucleotides [26]. Lgals1E2A-Cd274 was assembled by overlapping PCR and cloned into a Gateway entry vector using the pENTR/DTOPO Cloning Kit (Invitrogen). To convert the Rosa26 targeting vector into a destination plasmid, the AscI restriction site was used to insert a destination cassette for Gateway cloning with the Gateway Vector Conversion System (Invitrogen). Lgals1-E2A-Cd274 cDNA was then transferred into the destination cassette of the targeting vector using Gateway LR Clonase Enzyme Mix (Invitrogen).

\section{Microinjection of zygotes}

Cas9 mRNA was prepared in a single step by in vitro transcription from plasmid pCAG-Cas9-162A [14] linearized with AsiSI, AscI and XbaI using the mMessage mMachine T7 Ultra kit (Life Technologies, Ambion, AM1345, Life Technologies, Carlsbad, USA) (omitting the polyadenylation step) and the MEGAclear kit (Ambion, \#1908). To produce the template for sgRNA in vitro transcription, sgRosa26-1 was amplified by PCR 
from plasmid pX330-sgRosa26-1-T2A-BFP (Addgene \#64216) [13] with the forward primer T7-sgRosa26-for (5' -TTAATACGACTCACTATAGGACTCCAGTCTTT CTAGAAGAGT) and the reverse primer T7-sgRNArev (5'-AAAAGCACCGACTCGGTGCC). One microgram template DNA was used for in vitro transcription using the Megashortscript kit (Ambion, \#AM1354) followed by the MEGAclear kit for RNA purification. The quality of mRNAs was controlled by agarose gel electrophoresis under denaturing conditions using the NorthernMax-Gly system and the RNA Millenium size marker (Life Technologies). RNAs and targeting vectors were diluted in microinjection buffer $(10 \mathrm{mM}$ Tris, $0.1 \mathrm{mM}$ EDTA, $\mathrm{pH}$ 7.2) to the indicated working concentrations, filtrated through a centrifugal filter (Ultrafree, PFTE, Millipore, cat. no. UFC30LG25) and stored in single use aliquots at $-80{ }^{\circ} \mathrm{C}$. Where indicated, Cas 9 protein (ToolGen Inc, Seoul, South Corea) was supplemented immediately before injection. Step-by-step protocols for RNA and sample preparation are included as Supplementary Methods in the Additional file 2.

For microinjections, zygotes were obtained by mating of C57BL/6 N males with super-ovulated C57BL/6 N females (Charles River, Sulzbach, Germany) using standard procedures [27]. Zygotes were microinjected into one pronucleus as previously described [28]. Injected zygotes were transferred into the oviducts of pseudo-pregnant NMRI female mice to obtain live pups. All mice showed normal development and appeared healthy. Mice were handled according to institutional guidelines and all experiments were performed under registration and ethical approvement (Registration No. IC10b-G0162/ 12) by the Landesamt für Gesundheit and Soziales of the federal state of Berlin (Turmstr. 21, 10559 Berlin, Germany) Mice were housed in individually ventilated cages (IVC, Tecniplast) in a specific pathogen-free facility on a $12 \mathrm{~h}$ light/dark cycle with ad libitum access to food and water.

\section{PCR, T7EI and RLFP assays}

Genomic DNA from blastocysts was extracted using the QuickExtract DNA extraction kit (Epicentre) following the manufacturer's instructions. PCR was performed using Herculase II Fusion DNA Polymerase (Agilent Technology) with gene-specific primers. For the T7EI assay, the PCR product was cleaned up and digested with T7EI (New England Biolabs following the manufacturer's instructions. For the RLFP assay, PCR products were digested with the restriction enzyme XbaI (Thermo Scientific). Cleaved DNA fragments were separated on $2 \%$ agarose gels and the DNA concentration of each band was quantified using the ImageJ software. Percent values of indels were calculated as described [29]. For genotyping by PCR, serial primer pairs were used as listed below.

\section{DNA sequencing}

Specific PCR products were cleaned up and directly sequenced by the Sanger method (LGCgenomics, Berlin, Germany). In addition, the DNA fragments were cloned into pSTblue-1 Blunt vector (Novagen), plasmids were isolated using the NucleoSpin Plasmid kit (MachereyNagel). Plasmids were sequenced using T7 forward primer.

\section{Analysis of off-target sites}

The Rosa26-protospacer (ACTCCAGTCTTTCTAGAA GATGG) was aligned to the mouse genome ( $\mathrm{mm} 9)$ using BWA (0.7.12) [30]. Off-target sites were evaluated using an in-house developed tool for protospacer-design. The primers used for PCR-ampflication were P1 (5'TTGGTTCCCAACACTCACAG-3'), R1 (5' -TGTGTAA CTGCTCTGTTGTCTCC-3'), P2 (5'-CTTTGGGTTC CCTCAGTAGAAG-3'), R2 (5' -AAGACCCAAACAGG TATGCAG-3'), P3 (5'-CCACAGGGATAGGCAATA AAGA-3') and R3 (5'-GCTGAGCTGTCCCAATGA GT-3'). PCR products were sequenced by the Sanger method (LGCgenomics, Berlin, Germany) using R1, P2 and R3 for Off1, Off2 and Off3, respectively.

\section{Southern blotting}

Southern blotting for correct integration of the targeting construct into the Rosa26 locus was done as described [24]. Briefly, genomic DNA was isolated from tails and $10 \mu \mathrm{g}$ were digested with EcoRI. DNA fragments were separated on a $0.7 \%$ agarose gel. The gel was washed two times for $5 \mathrm{~min}$ in $\mathrm{dH}_{2} \mathrm{O}$, incubated two times for $15 \mathrm{~min}$ in $0.125 \mathrm{M} \mathrm{HCl}$ for depurination, washed two times for 5 min in $\mathrm{H}_{2} \mathrm{O}$, and was finally denatured by incubating two times for $15 \mathrm{~min}$ in $0.5 \mathrm{M} \mathrm{NaOH} / 1.5 \mathrm{M}$ $\mathrm{NaCl}$. DNA was blotted over night to a Hybond XL membrane (GE Healthcare). The membrane was then neutralized for $10 \mathrm{~min}$ in $0.5 \mathrm{M}$ Tris $-\mathrm{HCl} \mathrm{pH} 7.2 / 1 \mathrm{M}$ $\mathrm{NaCl}$, dried, and UV-crosslinked by irradiation with $120,000 \mu \mathrm{J} \mathrm{cm}^{-2}$. Then, the membrane was preincubated for $3 \mathrm{~h}$ at $65{ }^{\circ} \mathrm{C}$ in ExpressHyb Hybridization Solution (Takara). $25 \mathrm{ng}$ probe was radioactively labeled using Ladderman Labeling Kit (Takara) by adding $25 \mu \mathrm{Ci}{ }^{32} \mathrm{P}$-dCTP and purified on a Sephadex-G50 column (GE Healthcare). The probe was first denatured and then incubated with the membrane over night at $65{ }^{\circ} \mathrm{C}$. Next, the membrane was briefly washed twice in $2 \times \mathrm{SSC} / 1 \%$ SDS, then incubated in $2 \times \mathrm{SSC} / 1 \% \mathrm{SDS}$ for $30 \mathrm{~min}$, then incubated in $1 \times \mathrm{SSC} / 1 \% \mathrm{SDS}$ for $30 \mathrm{~min}$, followed by $0.5 \times \mathrm{SSC} / 1 \% \mathrm{SDS}$ for $30 \mathrm{~min}$, all at $65{ }^{\circ} \mathrm{C}$. The membrane was used to expose an X-ray film at $-80{ }^{\circ} \mathrm{C}$ in the dark for 3 to 7 days.

\section{B cell culture, stimulation and TAT-Cre treatment}

Naïve B cells from Rosa26 ${ }^{\mathrm{LSL}-\mathrm{Cas} 9}$ and C57BL/6 mice were isolated by $\mathrm{CD} 43$ depletion using $\mathrm{CD} 43$ microbeads 
(Miltenyi Biotec). B cells were cultured at $1 \times 10^{6}$ cells $/ \mathrm{ml}$ in DMEM medium supplied with $15 \% \mathrm{FBS}, 2 \mathrm{mM}$ HEPES (Gibco), $2 \mathrm{mM}$ Sodium Pyruvate (Gibco), $2 \mathrm{mM}$ L-Glutamine (Gibco), and 1x NAA (Gibco), betamercapthoethanol (Sigma) and stimulated with LPS $(10 \mu \mathrm{g} / \mathrm{ml})$. In addition, $5-10 \times 10^{6}$ naïve B cells isolated from Rosa $26^{\text {LSL-Cas9 }}$ mice were treated with TAT-Cre protein as previously described [31]. Briefly, CD43depleted B cells were washed 3 times with HyClone ${ }^{\mathrm{Tx}}$ ADCF-Mab medium (GE Heathcare), incubated with TAT-Cre for $45 \mathrm{~min}$ at $37{ }^{\circ} \mathrm{C}$, finally the cells were washed with complete medium. TAT-Cre-treated B cells were stimulated with LPS for 2 or 3 days.

\section{Retroviral transduction}

The MSCV plasmid expressing the sgRNA to target the mouse Rosa26 locus was transfected into the packaging cell line Plat-E (Cell Biolabs) using Calcium phosphate protocol. $24 \mathrm{~h}$ after transfection, the medium was changed and the transfected cells were incubated at $32{ }^{\circ} \mathrm{C}$. The viral supernatant was collected at 48 and $72 \mathrm{~h}$ after transfection. The supernatant was concentrated using Amicon Ultra-15 Centrifugal Filter (Merck) according to the manufacture's protocol. $5 \times 10^{5}$ of LPS-activated B cells were transduced with concentrated retroviral particles using spin transduction method. The reporter positive cells were quantified using a Fortessa cell analyzer (Becton Dickinson).

\section{FACS sorting and analysis}

The $\mathrm{BFP}^{+} \mathrm{B}$ cells were sorted into $15 \mathrm{ml}$ Falcon tubes with complete medium, cells were centrifuged and genomic DNA was isolated. For flow cytometry analysis, B cells were harvested, washed $2 x$ with cold PBS, resuspended in PBS/1 \% BSA FACS buffer and stained with anti-mouse CD19 Brilliant Violet $605^{\mathrm{m}}$ and B220 Brilliant Violet $785^{\mathrm{mix}}$ (Biolegend). The stained cells were analysed with a Fortessa cell analyzer (Becton Dickinson). Dead cells were excluded by DAPI (Sigma).

\section{Western blot analysis}

Naive B cells were treated with TAT-Cre protein, and stimulated with LPS $(10 \mu \mathrm{g} / \mathrm{ml})$ for 2 days. The protein lysates were isolated from the activated B cells as previously described [13] and separated by SDS-PAGE. Blots were probed with anti-Flag (M2, Sigma), antiCas9 (Novus Biologicals) and anti-beta-actin (AC-74, Sigma) antibodies. The probed blots were developed with secondary anti-mouse IgG HRP (eBioscience) and visualised using the ECL detection kit (GE Healthcare).

\section{Additional files}

Additional file 1: Figure S1. CRISPR/Cas9 induced DSBs at the Rosa26 intronic Xbal site in mouse zygotes; Figure S2: Sequence analysis of founder derived PCR products. Figure S3: Analysis of off-target activity. (DOC $1735 \mathrm{~kb}$ )

Additional file 2: Supplementary Methods and Plasmid maps. (PDF $358 \mathrm{~kb}$ )

\section{Abbreviations}

DSB: double-strand break; ES cells: embryonic stem cells; HDR: homology directed repair, LSL, lox-stop-lox; NHEJ: non-homologous end joining.

\section{Competing interests}

The authors declare that they have no competing interests.

\section{Authors' contributions}

VTC, TW, RG, RK performed and analysed experiments, VTC, TW, RG, KR, RK designed the project and wrote the paper. US, KP, TS and PV provided materials. All authors read and approved the final manuscript.

\section{Acknowledgements}

We thank Sabine Manz, Andrea Leschke, Patricia Kulig and Rainer Kabisch (Transgenics core facility) and Hans Peter Rahn (FACS core facility) for excellent technical support and are grateful to Dennis Kobelt (MDC) for cloning the pR26 AsiSI/Mlul vector. This work was supported by the European Research Council (ERC Advanced Grant 268921, to K.R.) and the German Ministry of Education and Research within the VIP program (TAL-CUT 03 V0261, to R.K.).

\section{Author details}

${ }^{1}$ Max-Delbrück-Center for Molecular Medicine, 13125 Berlin, Germany. ${ }^{2}$ Present Address: Bayer Pharma AG Building S107, 13353 Berlin, Germany. ${ }^{3}$ Harvard University, Cambridge, MA 02138, USA. ${ }^{4}$ Berlin Institute of Health, Kapelle-Ufer 2, 10117 Berlin, Germany.

Received: 30 July 2015 Accepted: 7 January 2016

Published online: 16 January 2016

\section{References}

1. Zambrowicz BP, Imamoto A, Fiering S, Herzenberg LA, Kerr WG, Soriano P. Disruption of overlapping transcripts in the ROSA beta geo 26 gene trap strain leads to widespread expression of beta-galactosidase in mouse embryos and hematopoietic cells. Proc Natl Acad Sci U S A. 1997;94: 3789-94.

2. Friedrich $G$, Soriano P. Promoter traps in embryonic stem cells: a genetic screen to identify and mutate developmental genes in mice. Genes Dev. 1991:5:1513-23.

3. Soriano P. Generalized lacZ expression with the ROSA26 Cre reporter strain. Nat Genet. 1999:21:70-1.

4. Capecchi MR. Gene targeting in mice: functional analysis of the mammalian genome for the twenty-first century. Nat Rev Genet. 2005;6:507-12.

5. Niwa H, Yamamura K, Miyazaki J. Efficient selection for high-expression transfectants with a novel eukaryotic vector. Gene. 1991;108:193-9.

6. Nagy A. Cre recombinase: the universal reagent for genome tailoring. Genesis. 2000;26:99-109.

7. Meyer M, de Angelis MH, Wurst W, Kuhn R. Gene targeting by homologous recombination in mouse zygotes mediated by zinc-finger nucleases. Proc Natl Acad Sci U S A. 2010;107:15022-6.

8. Wefers B, Ortiz O, Wurst W, Kühn R. Generation of targeted mouse mutants by embryo microinjection of TALENs. Methods San Diego Calif. Methods 2014:69:94-101.

9. Hsu PD, Lander ES, Zhang F. Development and applications of CRISPR-Cas9 for genome engineering. Cell. 2014;157:1262-78.

10. Wang $H$, Yang $H$, Shivalila CS, Dawlaty MM, Cheng AW, Zhang F, et al. One-step generation of mice carrying mutations in multiple genes by CRISPR/Cas-mediated genome engineering. Cell. 2013;153:910-8.

11. Singh P, Schimenti JC, Bolcun-Filas E. A mouse geneticist's practical quide to CRISPR applications. Genetics. 2015;199:1-15. 
12. Yang H, Wang H, Shivalila CS, Cheng AW, Shi L, Jaenisch R. One-step generation of mice carrying reporter and conditional alleles by CRISPR/ Cas-mediated genome engineering. Cell. 2013;154:1370-9.

13. Chu VT, Weber T, Wefers B, Wurst W, Sander S, Rajewsky K, et al. Increasing the efficiency of homology-directed repair for CRISPR-Cas9-induced precise gene editing in mammalian cells. Nat Biotechnol. 2015;33:543-8.

14. Brandl C, Ortiz O, Röttig B, Wefers B, Wurst W, Kühn R. Creation of targeted genomic deletions using TALEN or CRISPR/Cas nuclease pairs in one-cell mouse embryos. FEBS Open Bio. 2015;5:26-35.

15. Certo MT, Ryu BY, Annis JE, Garibov M, Jarjour J, Rawlings DJ, et al. Tracking genome engineering outcome at individual DNA breakpoints. Nat Methods. 2011;8:671-6.

16. Hartley JL, Temple GF, Brasch MA. DNA cloning using in vitro site-specific recombination. Genome Res. 2000;10:1788-95.

17. Maruyama T, Dougan SK, Truttmann MC, Bilate AM, Ingram JR, Ploegh HL. Increasing the efficiency of precise genome editing with CRISPR-Cas9 by inhibition of nonhomologous end joining. Nat Biotechnol. 2015;33:538-42.

18. Kasparek P, Krausova M, Haneckova R, Kriz V, Zbodakova O, Korinek V, et al. Efficient gene targeting of the Rosa26 locus in mouse zygotes using TALE nucleases. FEBS Lett. 2014;588:3982-8.

19. Ohtsuka M, Ogiwara S, Miura H, Mizutani A, Warita T, Sato M, et al. Pronuclear injection-based mouse targeted transgenesis for reproducible and highly efficient transgene expression. Nucleic Acids Res. 2010;38:e198.

20. Ohtsuka M, Miura H, Hayashi H, Nakaoka H, Kimura M, Sato M, et al. Improvement of pronuclear injection-based targeted transgenesis (PITT) by iCre mRNA-mediated site-specific recombination. Transgenic Res. 2013:22:873-5.

21. Platt RJ, Chen S, Zhou Y, Yim MJ, Swiech L, Kempton HR, et al. CRISPR-Cas9 knockin mice for genome editing and cancer modeling. Cell. 2014;159:440-55.

22. Kobayashi T, Kato-ltoh M, Yamaguchi T, Tamura C, Sanbo M, Hirabayashi M, et al. Identification of rat Rosa26 locus enables generation of knock-in rat lines ubiquitously expressing tdTomato. Stem Cells Dev. 2012;21:2981-6.

23. Li S, Flisikowska T, Kurome M, Zakhartchenko V, Kessler B, Saur D, et al. Dual fluorescent reporter pig for Cre recombination: transgene placement at the ROSA26 locus. PLoS One. 2014;9:e102455.

24. Srinivasan L, Sasaki Y, Calado DP, Zhang B, Paik JH, DePinho RA, et al. PI3 kinase signals BCR-dependent mature B cell survival. Cell. 2009;139:573-86.

25. Calado DP, Sasaki Y, Godinho SA, Pellerin A, Köchert K, Sleckman BP, et al. The cell-cycle regulator $\mathrm{c}-\mathrm{Myc}$ is essential for the formation and maintenance of germinal centers. Nat Immunol. 2012;13:1092-100.

26. Szymczak AL, Workman CJ, Wang Y, Vignali KM, Dilioglou S, Vanin EF, et al. Correction of multi-gene deficiency in vivo using a single "self-cleaving" 2A peptide-based retroviral vector. Nat Biotechnol. 2004;22:589-94.

27. Ittner LM, Götz J. Pronuclear injection for the production of transgenic mice. Nat Protoc. 2007:2:1206-15.

28. Wefers B, Panda SK, Ortiz O, Brandl C, Hensler S, Hansen J, et al. Generation of targeted mouse mutants by embryo microinjection of TALEN mRNA. Nat Protoc. 2013;8:2355-79.

29. Cong L, Ran FA, Cox D, Lin S, Barretto R, Habib N, et al. Multiplex genome engineering using CRISPR/Cas systems. Science. 2013;339:819-23.

30. Li H, Durbin R. Fast and accurate long-read alignment with BurrowsWheeler transform. Bioinforma Oxf Engl. 2010;26:589-95.

31. Nolden L, Edenhofer F, Haupt S, Koch P, Wunderlich FT, Siemen H, et al. Site-specific recombination in human embryonic stem cells induced by cell-permeant Cre recombinase. Nat Methods. 2006;3:461-7.

\section{Submit your next manuscript to BioMed Central and we will help you at every step:}

- We accept pre-submission inquiries

- Our selector tool helps you to find the most relevant journal

- We provide round the clock customer support

- Convenient online submission

- Thorough peer review

- Inclusion in PubMed and all major indexing services

- Maximum visibility for your research

Submit your manuscript at www.biomedcentral.com/submit

) Biomed Central 